\title{
High Throughput Random Access via Codes on Graphs: Coded Slotted ALOHA
}

\author{
Enrico Paolini, Gianluigi Liva, and Marco Chiani
}

\begin{abstract}
In this paper, coded slotted ALOHA (CSA) is introduced as a powerful random access scheme to the MAC frame. In CSA, the burst a generic user wishes to transmit in the MAC frame is first split into segments, and these segments are then encoded through a local a packet-oriented code prior to transmission. On the receiver side, iterative interference cancellation combined with decoding of the local code is performed to recover from collisions. The new scheme generalizes the previously proposed irregular repetition slotted ALOHA (IRSA) technique, based on a simple repetition of the users' bursts. An interpretation of the CSA interference cancellation process as an iterative erasure decoding process over a sparse bipartite graph is identified, and the corresponding density evolution equations derived. Based on these equations, asymptotically optimal CSA schemes are designed for several rates and their performance for a finite number of users investigated through simulation and compared to IRSA competitors. Throughputs as high as 0.8 are demonstrated. The new scheme turns out to be a good candidate in contexts where power efficiency is required.
\end{abstract}

\section{INTRODUCTION}

Although demand assignment multiple access (DAMA) medium access control (MAC) protocols guarantee an efficient usage of the available bandwidth [1], MAC random access schemes remain an appealing and popular solution for wireless networks. Among them, slotted ALOHA (SA) [1]-[3] is currently adopted as the initial access scheme in both cellular terrestrial and satellite communication networks [4]. In [5] an improvement to SA was proposed, namely, diversity slotted ALOHA (DSA). In DSA, each packet (also called burst) is transmitted twice over the MAC frame, which provides a slight throughput gain over SA. As a drawback, for the same peak transmission power of the SA scheme, the average transmitted power of DSA is doubled.

A more effective use of the burst repetition is provided by contention resolution diversity slotted ALOHA (CRDSA) [6], whose basic idea is the adoption of interference cancellation (IC) to resolve collisions. More specifically, with respect to DSA, each of the twin replicas of a burst, transmitted within a MAC frame, possesses a pointer to the slot position where the respective copy was sent. Whenever a clean burst is detected and successfully decoded, the pointer is extracted and the interference contribution caused by the burst copy on the corresponding slot is removed. This procedure is iterated,

E. Paolini and M. Chiani are with DEIS/WiLAB, University of Bologna, via Venezia 52, 47521 Cesena (FC), Cesena, Italy (e-mail: e.paolini@unibo.it, marco.chiani@unibo.it).

Gianluigi Liva is with German Aerospace Center (DLR), D-82234 Wessling, Germany (e-mail: Gianluigi.Liva@dlr.de).

This work was supported in part by the EC under Seventh FP grant agreement ICT OPTIMIX n.INFSO-ICT-214625. possibly allowing to recover the whole set of bursts transmitted within the same MAC frame. This results in a remarkably improved normalized throughput $S$ (defined as the probability of successful packet transmission per time slot) which may reach $S \simeq 0.55$, whereas the peak throughput for pure $\mathrm{SA}$ is $S=1 / e \simeq 0.37$. Further improvements can be achieved by exploiting the capture effect [3], [7].

In [8], [9] irregular repetition slotted ALOHA (IRSA) was introduced to provide a further throughput gain over CRDSA. A higher normalized throughput is achieved by IRSA by allowing a variable and judiciously designed repetition rate for each burst. As for DSA, the performance improvement achieved by CRDSA/IRSA has a counterpart in the increment of the average transmitted power. Since CRDSA is a specific instance of IRSA, in the following we will refer in general to IRSA. In [8] it is also illustrated how the iterative burst recovery process on the receiver side can be represented via a bipartite graph and how, under the assumption of an ideal channel estimation and of a sufficiently large signal-tonoise ratio (SNR), it shares several commonalities with the graph representation of the erasure recovery process of modern channel codes on sparse graphs [10], [11].

In this paper, we introduce a further generalization of IRSA, named coded slotted ALOHA (CSA). The basic idea of CSA is to encode (instead of simply repeat) bursts using local codes prior to transmission in the MAC frame and to combine, on the receiver side, iterative IC with decoding of the local codes to recover from collisions. The new scheme turns out to be interesting especially in contexts where power efficiency is required. Density evolution equations for CSA are derived to analyze the IC process in an asymptotic setting, leading to the calculation of the peak asymptotic throughput. Numerical results are then presented to illustrate the validity of the proposed asymptotic analysis and its effectiveness in the design of CSA access schemes for a finite number of users.

\section{SYSTEM MODEL}

Similarly to [6], [8], we consider a random access scheme where the slots are grouped in MAC frames, all with the same length (in slots). We further restrict to the case where each user attempts one burst transmission per MAC frame.

Consider $M$ users, each attempting the transmission of a burst of time duration $T_{\mathrm{SA}}$ over a MAC frame of time duration $T_{\mathrm{F}}$. Neglecting guard times, the MAC frame is composed of $N_{\mathrm{SA}}=T_{\mathrm{F}} / T_{\mathrm{SA}}$ slots. In $\mathrm{SA}$, each user would independently choose one of the $N_{\mathrm{SA}}$ slots uniformly at random and would attempt transmission of his burst into that slot. In IRSA each 
user would generate a certain number $r$ of replicas of his burst, where $r$ may be not the same for two different users, and would transmit the $r$ replicas into $r$ slots chosen uniformly at random among the available $N_{\mathrm{SA}}$ slots.

In CSA, when a user wishes to transmit a burst of time duration $T_{\mathrm{SA}}$ over the MAC frame, the burst is divided into $k$ information sub-bursts (also called information segments), each of time duration $T_{\mathrm{CSA}}=T_{\mathrm{SA}} / k$. The $k$ information segments are then encoded by the user via a packet-oriented binary linear block code which generates $n_{h}$ encoded segments, each of time duration $T_{\mathrm{CSA}}=T_{\mathrm{SA}} / k$. For each transmission, the code to be employed is drawn randomly by the user from a set of $n_{c}$ possible codes. For $h \in\left\{1, \ldots, n_{c}\right\}$ the $h$ th code, denoted by $\mathscr{C}_{h}$, is a $\left(n_{h}, k, d_{\min }^{(h)}\right)$ code, that is it has length $n_{h}$, dimension $k$, and minimum distance $d_{\min }^{(h)}$. We further impose that $\mathscr{C}_{h}$ has no idle bits and fulfills $d_{\text {min }}^{(h)} \geq 2$. We assume that, at any transmission, each user independently chooses his local code according to a probability mass function (p.m.f.) $\boldsymbol{P}=\left[P_{h}\right]_{h=1}^{n_{c}}$ which is the same for all users. Denoting again by $T_{\mathrm{F}}$ the MAC frame duration, the MAC frame is composed of $N_{\mathrm{CSA}}=T_{\mathrm{F}} / T_{\mathrm{CSA}}=k N_{\mathrm{SA}}$ slots. The $n_{h}$ coded segments are then transmitted by the user over $n_{h}$ slots picked uniformly at random. Note that IRSA may be seen as a special case of CSA where $k=1$ and each $\mathscr{C}_{h}$ is a repetition code of length $n_{h}$, and that SA is a special case of IRSA where $n_{h}=1$ for all users 1 The overall rate of CSA is defined as $R=k / \bar{n}$, where $\bar{n}:=\sum_{h=1}^{n_{c}} P_{h} n_{h}$ is the expected length of the code employed by the generic user. Note that $\Delta P=\bar{n} / k=1 / R$ represents the increment of average power with respect to pure SA.

It is now convenient to introduce a graph representation of CSA, depicted in Fig. 1 Let us consider a MAC frame composed of $N_{\mathrm{CSA}}$ slots, in which $M$ users attempt a transmission. The MAC frame status can be represented by a bipartite graph, $\mathcal{G}=(\mathcal{B}, \mathcal{S}, \mathcal{E})$, consisting of a set $\mathcal{B}$ of $M$ burst nodes (one for each burst transmitted in the MAC frame), a set $\mathcal{S}$ of $N_{\mathrm{CSA}}$ sum nodes (one for each slot), and a set $\mathcal{E}$ of edges. An edge connects a burst node (BN) $b_{i} \in \mathcal{B}$ to a sum node (SN) $s_{j} \in \mathcal{S}$ if and only if an encoded segment associated with the $i$ th burst is transmitted in the $j$ th slot. In other words, BNs correspond to bursts, SNs to slots, and edges to encoded segments. Therefore, a burst split into $k$ information segments and encoded via the code $\mathscr{C}_{h}$ is represented as a BN with $n_{h}$ neighbors. Correspondingly, a slot where $d$ replicas collide is represented as a $\mathrm{SN}$ with $d$ connections. The number of edges emanating from a node is the node degree. Moreover, a $\mathrm{BN}$ where $\mathscr{C}_{h}$ is employed during the current transmission is referred to as a $\mathrm{BN}$ of type $h$.

In our analysis, we rely on three assumptions. 1) Sufficiently high SNR. This allows to claim that, when a segment is received in a clean slot, it is known at the receiver. 2) Ideal channel estimation. Under this assumption (and the previous one), ideal IC is possible, allowing the recovery of collided bursts with a probability that is essentially one. 3) Destructive collisions. Segments that collide in a slot are treated as erasures. These assumptions simplify the analysis

\footnotetext{
${ }^{1}$ We point out that CSA may be seen as a generalization also of the schemes proposed in [12], [13], where no IC was used.
}

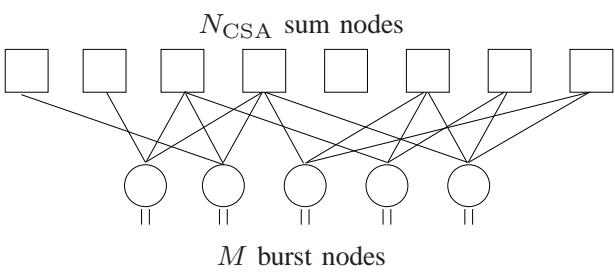

Fig. 1. Graph representation of CSA. Circles are the burst nodes and represent the $M$ users, squares are the sum nodes and represent the $N$ slots. The degree of a burst node is equal to the length of the locally employed code. The degree of a sum node is equal to the number of collided encoded segments. The example is for $k=2$.

without substantially affecting the obtained results, as shown in [6] and [9] for CRDSA and IRSA, respectively.

Each coded segment associated with a BN of type $h$ is equipped with information about the relevant user and with a pointer to the other $n_{h}-1$ segments 2 On the receiver side, segments which collided in some slot with those sent by another user are marked as lost, so that a $\mathrm{BN}$ is connected to "known" edges and to "unknown" ones. Hence, some of its information bursts are known, and the others unknown. At the generic BN (say of type $h$ ), erasure decoding of the code $\mathscr{C}_{h}$ may allow to recover some of the unknown encoded and information segments. It is now possible to subtract the interference contribution of the newly recovered encoded segments from the signal received in the corresponding slot. If $d-1$ segments that collided in a SN of degree $d$ have been recovered by the corresponding BNs, the remaining segment becomes known. The IC process combined with local decoding at the BNs proceeds iteratively, i.e., cleaned segments may allow solving other collisions. Note that this procedure is equivalent to iterative decoding of a doubly-generalized low-density paritycheck (D-GLDPC) code over the erasure channel [14], where variable nodes are generic linear block codes and check nodes are single parity-check (SPC) codes.

Denoting by $N=N_{\mathrm{CSA}}$ the number of slots (a multiple of $k$ ), the logical normalized offered traffic $G$ is given by

$$
G=\frac{k M}{N}
$$

The normalized throughput $S$ is defined as the probability of successful packet transmission per time slot. For example, for standard SA we have $S=G e^{-G}$.

Finally, we recall the definition of information function of a linear block code [15]. Let $\mathbf{G}$ be a generator matrix for an $(n, k)$ linear block code $\mathscr{C}$. The $g$ th un-normalized information function of $\mathscr{C}$, denoted by $\tilde{e}_{g}$, is defined as the summation of the ranks over all the possible submatrices obtained selecting $g$ columns (with $0 \leq g \leq n$ ) out of $\mathbf{G}$.

\footnotetext{
${ }^{2}$ In practical implementations, the overhead due to the inclusion of pointers in the segment header may be reduced by adopting more efficient techniques. For fixed $k$, one may include in the segment header the code index $h$ together with a random seed, out of which it is possible to reconstruct (by a pre-defined pseudo-random number generator) the positions of the $n_{h}$ segments.

${ }^{3}$ In CSA and IRSA we distinguish the logical load $G$ from the physical load given by $\frac{\bar{n}}{k} G=G / R$ and representing the average number of transmitted segments per slot. The logical load $G$ provides a direct measure of the traffic handled by the scheme. Note that the two concepts coincide in pure SA.
} 


\section{Density EVolution, Threshold, AND Stability}

The degree distribution of the SNs from a node perspective is defined as

$$
\Psi(x)=\sum_{d \geq 0} \Psi_{d} x^{d}
$$

where $\Psi_{d}$ is the probability that a SN has degree $d$.

Let us consider a user encoding his segments through the code $\mathscr{C}_{h}$, and allocating his $n_{h}$ encoded segments into $n_{h}$ slots chosen randomly. Then, the probability that the BN associated with this user (say $U$ ) is connected to a SN $A$ may be expressed as the ratio between the number of ways of connecting the $n_{h}$ sockets of $U$ to the $N$ SNs such that $U$ is connected to $A$, to the total number of ways of connecting the $n_{h}$ sockets of $U$ to the $N$ SNs: $\operatorname{Pr}\left\{U\right.$ is connected to $A \mid U$ uses $\left.\mathscr{C}_{h}\right\}=\left(\begin{array}{c}N-1 \\ n_{h}-1\end{array}\right) /\left(\begin{array}{c}N \\ n_{h}\end{array}\right)=\frac{n_{h}}{N}$. Therefore we have:

$$
\operatorname{Pr}\{U \text { is connected to } A\}=\sum_{h=1}^{n_{c}} P_{h} \frac{n_{h}}{N}=\frac{\bar{n}}{N} .
$$

Since each user selects his slots independently of all the other users, the probability $\Psi_{d}$ that a SN has degree $d$ (that is the probability that the $\mathrm{SN}$ is chosen by $d$ users) is given by

$$
\begin{aligned}
\Psi_{d} & =\left(\begin{array}{c}
M \\
d
\end{array}\right)\left(\frac{\bar{n}}{N}\right)^{d}\left(1-\frac{\bar{n}}{N}\right)^{M-d} \\
& \stackrel{\text { a) }}{=}\left(\begin{array}{c}
M \\
d
\end{array}\right)\left(\frac{\bar{n} G}{k M}\right)^{d}\left(1-\frac{\bar{n} G}{k M}\right)^{M-d} \\
& \rightarrow \frac{e^{-\frac{\bar{n}}{k} G}}{d !}\left(\frac{\bar{n}}{k} G\right)^{d} \quad \text { as } M \rightarrow \infty
\end{aligned}
$$

where equality (a) follows from (11). Therefore, in the limit where $M$ (and consequently, for fixed $G$ and $k, N$ through (11) tends to infinity, (2) may be written as

$$
\Psi(x)=\sum_{d \geq 0} \frac{e^{-\frac{\bar{n}}{k} G}}{d !}\left(\frac{\bar{n}}{k} G\right)^{d} x^{d}=\exp \left(-\frac{\bar{n}}{k} G(1-x)\right) .
$$

Using (4) we can now express the probability $\rho_{d}$ that an edge is connected to a $\mathrm{SN}$ of degree $d \geq 1$ as:

$$
\rho_{d}=\frac{\Psi_{d} d}{\sum_{i \geq 1} \Psi_{i} i}=\frac{\Psi_{d} d}{\Psi^{\prime}(1)}=\frac{\left(\frac{\bar{n}}{k} G\right)^{d-1}}{(d-1) !} e^{-\frac{\bar{n}}{k} G} .
$$

Therefore, the degree distribution of the SNs from an edge perspective is given by

$$
\rho(x)=e^{-\frac{\bar{n}}{k} G} \sum_{d \geq 1} \frac{\left(\frac{\bar{n}}{k} G x\right)^{d-1}}{(d-1) !}=\exp \left(-\frac{\bar{n}}{k} G(1-x)\right)
$$

and $\rho(x)=\Psi(x)$.

For given $k$ and $G$, we investigate the evolution of the decoding process described in Section $\Pi$ in the asymptotic case where $M \rightarrow \infty$ (and consequently $N \rightarrow \infty$ through (1)).

Proposition 1: Assume MAP decoding is used at each $\mathrm{BN}$. At the $i$ th decoding iteration, let $p_{i-1}$ be the average probability that an edge carries an erasure messag 4 from the

\footnotetext{
${ }^{4}$ This is the probability that an edge is associated with an encoded segment that is still unknown.
}

SNs to the BNs. Consider a BN where $\mathscr{C}_{h}$ is employed and let $q_{i}^{(h)}$ be the average probability that an edge carries an erasure message outgoing from the BN, after MAP decoding at the $\mathrm{BN}$. Then, we have

$$
\begin{aligned}
q_{i}^{(h)}= & \frac{1}{n_{h}} \sum_{t=0}^{n_{h}-1} p_{i-1}^{t}\left(1-p_{i-1}\right)^{n_{h}-1-t}\left[\left(n_{h}-t\right) \tilde{e}_{n_{h}-t}^{(h)}\right. \\
& \left.-(t+1) \tilde{e}_{n_{h}-1-t}^{(h)}\right]
\end{aligned}
$$

where $\tilde{e}_{g}^{(h)}$ is the $g$ th unnormalized information function of $\mathscr{C}_{h}$.

The proof of Proposition 1 is omitted due to space constraints. Note that the proof follows exactly the same argument used in [16, Theorem 2] to derive the expression of the EXIT function of a linear block code without idle bits over the binary erasure channel.

Proposition 2: Assume MAP decoding is used at each BN. At the $i$ th iteration, let $p_{i-1}$ be the average probability that an edge carries an erasure message from the SNs to the BNs, before MAP decoding at the burst node. Let $q_{i}$ be the average probability that an edge carries an erasure message from the burst nodes to the SNs, after MAP decoding at the BNs. Then:

$$
\begin{aligned}
q_{i}= & \frac{1}{\bar{n}} \sum_{h=1}^{n_{c}} P_{h} \sum_{t=0}^{n_{h}-1} p_{i-1}^{t}\left(1-p_{i-1}\right)^{n_{h}-1-t}\left[\left(n_{h}-t\right) \tilde{e}_{n_{h}-t}^{(h)}\right. \\
& \left.-(t+1) \tilde{e}_{n_{h}-1-t}^{(h)}\right] .
\end{aligned}
$$

Proof: For all $h \in\left\{1, \ldots, n_{c}\right\}$, let $\lambda_{h}$ be the probability that an edge is connected to a $\mathrm{BN}$ of type $h$. We have

$$
\lambda_{h}=\frac{P_{h} n_{h}}{\bar{n}} .
$$

The proposition is proved by observing that

$$
q_{i}=\sum_{h=1}^{n_{c}} \lambda_{h} q_{i}^{(h)}
$$

and by incorporating (7) and (9) into (10).

The following is a well-known result from basic density evolution on the erasure channel for irregular LDPC codes [11].

Proposition 3: At the $i$ th iteration, let $q_{i}$ be the average probability that an edge carries an erasure message from the BNs to the SNs before decoding at the SNs. Let $p_{i}$ be the average probability that an edge carries an erasure message from the SNs to the BNs after IC at the SNs. Then:

$$
p_{i}=1-\rho\left(1-q_{i}\right) .
$$

Incorporating (8) into (11) and recalling (6), we obtain the nonlinear difference equation

$$
\begin{aligned}
p_{i}=1-\exp \{ & -\frac{G}{k} \sum_{h=1}^{n_{c}} P_{h} \sum_{t=0}^{n_{h}-1} p_{i-1}^{t}\left(1-p_{i-1}\right)^{n_{h}-1-t} \\
& \left.\times\left[\left(n_{h}-t\right) \tilde{e}_{n_{h}-t}^{(h)}-(t+1) \tilde{e}_{n_{h}-1-t}^{(h)}\right]\right\}
\end{aligned}
$$

which expresses the evolution of the average probability that an edge carries an erasure message at the $i$ th decoding 
iteration. The initial value of 12 shall be set to $p_{0}=1$. The asymptotic threshold $G^{*}$ of the system is defined as

$$
G^{*}:=\sup \left\{G \geq 0: p_{i} \rightarrow 0 \text { as } i \rightarrow \infty, p_{0}=1\right\} .
$$

The threshold $G^{*}$ is the supremum $G$ such that, in the asymptotic setting $M \rightarrow \infty$, the normalized throughput $S$ fulfills $S=G$. For all values of $G<G^{*}$ the offered traffic turns into useful throughput and therefore $G^{*}$ is the asymptotic peak throughput.

Using standard bifurcation theory, the threshold $G^{*}$ is equal to the smallest $G>0$ such that, for some $0 \leq x<1,(x, G)$ is a solution to the system of simultaneous equations

$$
\begin{aligned}
f(x, G) & =x \\
\frac{\partial f(x, G)}{\partial x} & =1
\end{aligned}
$$

where

$$
\begin{aligned}
f(x, G):=1-\exp \{ & -\frac{G}{k} \sum_{h=1}^{n_{c}} P_{h} \sum_{t=0}^{n_{h}-1} a_{t}^{(h)} \\
& \left.\times x^{t}(1-x)^{n_{h}-1-t}\right\}
\end{aligned}
$$

and $a_{t}^{(h)}:=\left(n_{h}-t\right) \tilde{e}_{n_{h}-t}^{(h)}-(t+1) \tilde{e}_{n_{h}-1-t}^{(h)}$.

\section{A. Stability}

Difference equations such as (12) are often used to model discrete dynamical systems. These systems are typically analyzed as regard to the stability of their fixed (or steadystate equilibrium) points. A fixed point $\hat{x}$ of $x_{\ell}=f\left(x_{\ell-1}\right)$ is known to be locally stable if there exists $\epsilon>0$ such that $\lim _{\ell \rightarrow \infty} x_{\ell}=\hat{x}$ for all $x_{0}$ such that $\left|x_{0}-\hat{x}\right|<\epsilon$. The following well-known result establishes a necessary and sufficient condition for local stability of a fixed point.

Lemma 1: A fixed point $\hat{x}$ of a discrete dynamical system $x_{\ell}=f\left(x_{\ell-1}\right)$, where $f: \mathbb{R} \mapsto \mathbb{R}$ is a differentiable and singlevalued function, is locally stable if and only if $\left|f^{\prime}(\hat{x})\right|<1$.

It is readily shown that $p=0$ is a fixed point of (12), corresponding to successful IC. Therefore we may apply Lemma 1 to study its stability. We obtain the following result.

Proposition 4 (Stability condition): For $h \in\left\{1, \ldots, n_{c}\right\}$, let $\mathscr{C}_{h}$ be a $\left(n_{h}, k, d_{\text {min }}^{(h)}\right)$ linear block code employed by each user with probability $P_{h}$ at each transmission and $A_{w}^{(h)}$ be the number of weight- $w$ codewords of $\mathscr{C}_{h}$. Moreover, let $\underline{d}=\min _{h}\left\{d_{\min }^{(h)}\right\}$ and $\mathcal{D}=\left\{h: d_{\min }^{(h)}=\underline{d}\right\}$. If $\underline{d}=2$, then the fixed point $p=0$ of 12 is locally stable if and only if

$$
G<\frac{k}{2 \bar{A}_{2}}
$$

where $\bar{A}_{2}=\sum_{h \in \mathcal{D}} P_{h} A_{2}^{(h)}$ is the average number of weight-2 codewords. Else, if $\underline{d} \geq 3$, then the fixed point $p=0$ of 12 is stable for any value of $G$.

Proof: Let us define again $f(x, G)$ as in (15) and let us denote by $\mathcal{S}_{g}^{(h)}$ the generic $(k \times g)$ matrix obtained by selecting $g$ columns in (any representation of) the generator matrix of
$\mathscr{C}_{h}$, irrespective of the order of the $g$ columns, and by $\sum_{\mathcal{S}_{g}^{(h)}}$ the summation over all $\left(\begin{array}{l}n \\ g\end{array}\right)$ matrices $\mathcal{S}_{g}^{(h)}$. We have:

$$
\begin{aligned}
f^{\prime}(0) & =\frac{G}{k} \sum_{h=1}^{n_{c}} P_{h} a_{1}^{(h)} \\
& =\frac{2 G}{k} \sum_{h=1}^{n_{c}} P_{h}\left[\frac{\left(n_{h}-1\right) \tilde{e}_{n-1}^{(h)}}{2}-\tilde{e}_{n-2}^{(h)}\right] \\
& \stackrel{(\text { a) }}{=} \frac{2 G}{k} \sum_{h=1}^{n_{c}} P_{h}\left[k\left(\begin{array}{c}
n_{h} \\
n_{h}-2
\end{array}\right)-\tilde{e}_{n-2}^{(h)}\right] \\
& =\frac{2 G}{k} \sum_{h=1}^{n_{c}} P_{h} \sum_{\mathcal{S}_{n-2}^{(h)}}\left(k-\operatorname{rank}\left(\mathcal{S}_{n-2}^{(h)}\right)\right) \\
& \stackrel{\text { (b) }}{=} \begin{cases}\frac{2 G}{k} \bar{A}_{2} & \text { if } \quad \underline{d}=2 \\
0 & \text { if } \quad \underline{d} \geq 3\end{cases}
\end{aligned}
$$

In the previous equation list, both (a) and (b) rely on the hypothesis that $d_{\min } \geq 2$ and on [17, Proposition 2].

The stability condition is a necessary, but in general not sufficient condition for successful decoding. Note also that the stability condition implies

$$
G^{*} \leq \frac{k}{2 \bar{A}_{2}} .
$$

that will be referred to as the stability upper bound, denoted by $G_{\mathrm{sb}}^{*}$. Note that in the IRSA case $(k=1)$ we have $\bar{A}_{2}=P_{2}$, where $P_{2}$ is the probability to select the length- 2 repetition code, and therefore for IRSA we obtain $G^{*} \leq \frac{1}{2 P_{2}}$.

In the case where $\underline{d}=2,(18)$ may be achieved with equality. Indeed, this is the case when $n_{c}=1$ and the binary linear block code $\mathscr{C}$ employed by all users is a SPC code.

Proposition 5: Let $n_{c}=1$ and the linear block code $\mathscr{C}$ employed by all users be a $(k+1, k)$ SPC code. Then

$$
G^{*}=\frac{1}{k+1}
$$

and (18) is achieved with equality.

The proof is easily obtained by recasting (13) and (14) for the special case of SPC codes and by showing that $(x, G)=$ $\left(0, \frac{1}{n}\right)$ is a solution to the system and that no $G<\frac{1}{n}$ exists such that $(x, G)$ is a solution to the system for any $0 \leq x<1$.

\section{CSA FROM RANDOM LINEAR BLOCK CODES}

So far the generic user has been assumed to encode, at each transmission, its $k$ information segments via an $\left(n_{h}, k, d_{\min }^{(h)}\right)$ binary linear block code picked randomly with p.m.f. $\boldsymbol{P}=$ $\left[P_{h}\right]_{h=1}^{n_{c}}$ from an ensemble of $n_{c}$ candidate codes. In this section, we consider a slightly different situation. Specifically, we assume that, at each transmission, the generic user picks randomly a codeword length $n_{s}>k$ from the ensemble $\left\{n_{1}, \ldots, n_{s_{\max }}\right\}$ with p.m.f. $\boldsymbol{Q}=\left[Q_{n_{s}}\right]_{s=1}^{s_{\max }}$ and encodes his $k$ segments through a binary $\left(k \times n_{s}\right)$ generator matrix generated uniformly at random from the set of all rank- $k\left(k \times n_{s}\right)$ binary matrices representing $\left(n_{s}, k\right)$ linear block codes without idle bits and with minimum distance at least 2 . We are interested in calculating the expected threshold $\bar{G}^{*}$ for this scheme. The 
TABLE I

OPTIMIZED PROBABILITY DISTRIBUTION $\boldsymbol{P}$ FOR IRS A SCHEMES WITH RATES $1 / 3,2 / 5$ AND $R=1 / 2$ AND OPTIMIZED PROBABILITY DISTRIBUTION $\boldsymbol{Q}$ FOR CSA SCHEMES WITH $k=2$ AND RATES $1 / 3,2 / 5,1 / 2$ AND $3 / 5$ UNDER THE RANDOM CODE HYPOTHESIS.

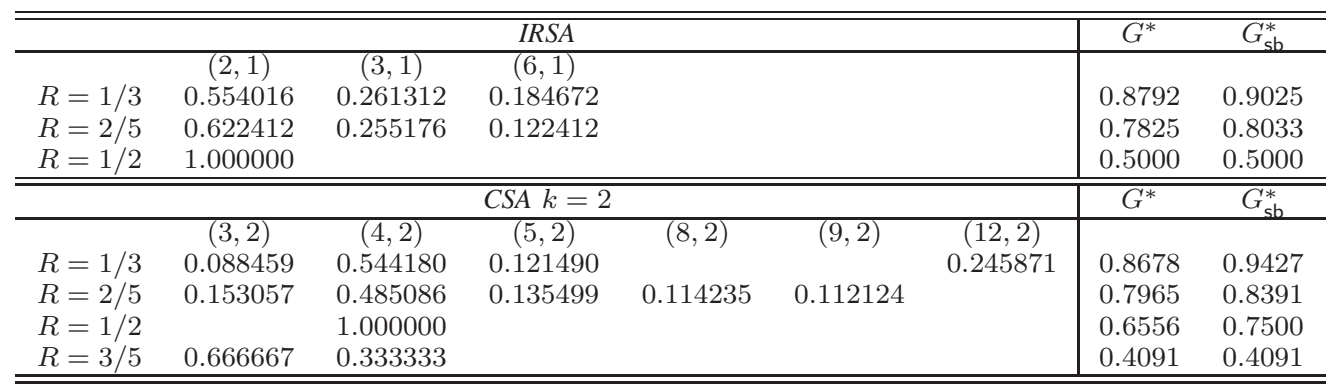

advantage of a random code hypothesis is to allow to release the analysis from considering a specific set of $n_{c}$ codes.

With respect to the previous case, the expression (4) of $\Psi(x)$ and the expression (6) of $\rho(x)$ remain unchanged, provided the definition of $\bar{n}$ is updated as $\bar{n}=\sum_{s=1}^{s_{\max }} Q_{n_{s}} n_{s}$. Analogously, (11) is not affected by the random code hypothesis. On the other hand, we now update (8) by replacing $q_{i}$ with its average value $\bar{q}_{i}$. Denoting by $\mathrm{G}_{\left(n_{s}, k\right)}$ the ensemble of all rank- $k(k \times$ $n_{s}$ ) binary matrices representing linear block codes without idle bits and with minimum distance at least 2 , and by $\mathbb{E}_{\mathrm{G}_{\left(n_{s}, k\right)}}$ the expectation operator over $\mathrm{G}_{\left(n_{s}, k\right)}$, we have

$$
\begin{aligned}
\bar{q}_{i} & =\frac{1}{\bar{n}} \sum_{s=1}^{s_{\max }} Q_{n_{s}} \sum_{t=0}^{n_{s}-1} p_{i-1}^{t}\left(1-p_{i-1}\right)^{n_{s}-1-t} \\
& \times\left[\left(n_{s}-t\right) \mathbb{E}_{\mathrm{G}_{\left(n_{s}, k\right)}}\left(\tilde{e}_{n_{s}-t}\right)-(t+1) \mathbb{E}_{\mathrm{G}_{\left(n_{s}, k\right)}}\left(\tilde{e}_{n_{s}-1-t}\right)\right]
\end{aligned}
$$

where again $\bar{n}=\sum_{s=1}^{s_{\max }} Q_{n_{s}} n_{s}$. The expectation $\mathbb{E}_{\mathrm{G}_{\left(n_{s}, k\right)}}\left(\tilde{e}_{g}\right)$ may be calculated using the following result developed in [18], where a recursive technique to calculate the functions $J(k, n, k)$ and $K(k, n, g, u, k)$ is also available.

Proposition 6: For given positive integers $n, k<n$, and $g \leq n, \mathbb{E}_{\mathrm{G}_{(n, k)}}\left(\tilde{e}_{g}\right)$ is given by

$$
\mathbb{E}_{\mathrm{G}_{(n, k)}}\left(\tilde{e}_{g}\right)=\left(\begin{array}{l}
n \\
g
\end{array}\right) \sum_{u=1}^{\min \{k, g\}} u \frac{K(k, n, g, u, k)}{J(k, n, k)}
$$

where $J(k, n, k)$ is the number of rank- $k(k \times n)$ binary matrices without zero columns and without independent columns, and where $K(k, n, g, u, k)$ is the number of rank- $k(k \times n)$ binary matrices without zero columns, without independent columns and such that the first $g$ columns have rank $u$

The average threshold $\bar{G}^{*}$ may be calculated by properly updating the simultaneous equations (13) and (14). Specifically, defining the function $\bar{f}(x)$ as

$$
\begin{aligned}
\bar{f}(x, G):=1-\exp \{ & -\frac{G}{k} \sum_{s=1}^{s_{\max }} Q_{n_{s}} \sum_{t=0}^{n_{s}-1} \mathbb{E}_{\mathrm{G}_{n_{s}, k}}\left(a_{t}\right) \\
& \left.\times x^{t}(1-x)^{n_{s}-1-t}\right\}
\end{aligned}
$$

\footnotetext{
${ }^{5}$ In this context, a column is said to be independent when deleting the column from the matrix does not affect the rank of the matrix.
}

where $\mathbb{E}_{\mathrm{G}_{n_{s}, k}}\left(a_{t}\right)=\left[\left(n_{s}-t\right) \mathbb{E}_{\mathrm{G}_{\left(n_{s}, k\right)}}\left(\tilde{e}_{n_{s}-t}\right)-(t+1)\right.$ $\left.\mathbb{E}_{\mathrm{G}_{\left(n_{s}, k\right)}}\left(\tilde{e}_{n_{s}-1-t}\right)\right], \bar{G}^{*}$ is equal to the smallest $G>0$ such that, for some $0 \leq x<1,(x, G)$ is a solution of (13) and (14), where now $f(x, G)$ is replaced by $\bar{f}(x, G)$.

Using a proof technique analogous to that of Proposition 4. it is easy to show that the stability bound is still given by (18), where now $\bar{A}_{2}=\sum_{s=1}^{s_{\max }} Q_{n_{s}} \bar{A}_{2}^{\left(n_{s}, k\right)}$ and

$$
\bar{A}_{2}^{\left(n_{s}, k\right)}=\left(\begin{array}{c}
n_{s} \\
2
\end{array}\right)\left(k-\sum_{u=1}^{\min \left\{k, n_{s}-2\right\}} u \frac{K\left(k, n_{s}, n_{s}-2, u, k\right)}{J\left(k, n_{s}, k\right)}\right)
$$

is the expected number of weight-2 codewords of an $\left(n_{s}, k\right)$ linear block code picked uniformly at random in the ensemble of all $\left(n_{s}, k\right)$ linear block codes without idle bits and with minimum distance at least 2 .

\section{NumericAl Threshold OPTIMIZATION AND COMPARISON WITH IRSA}

The analysis tool developed in Section $\coprod$ allows to calculate the threshold for a given choice of the $n_{c}$ linear block codes $\mathscr{C}_{h}, h \in\left\{1, \ldots, n_{c}\right\}$, and of the p.m.f. $\boldsymbol{P}$. Analogously, the tool developed in Section IV allows to evaluate the threshold of a CSA scheme under the random code hypothesis, for a given choice of the $s_{\max }$ lengths $n_{s}, n \in\left\{1, \ldots, s_{\max }\right\}$, and of the p.m.f. $Q$. These tools can be exploited to derive optimal (in the sense of maximizing the threshold $G^{*}$ ) probability distributions $\boldsymbol{P}$ and $\boldsymbol{Q}$ in the two cases.

Some optimized distribution profiles are shown in Table I] Among the several possible algorithms available to find the global maximum of a nonlinear function, differential evolution [19] has been used. In the upper part of the table, optimized probability distributions $\boldsymbol{P}$ are reported for an IRSA scheme with rates $1 / 2,2 / 5$ and $1 / 3$, while in the lower part optimized probabilities distributions $Q$ are illustrated for a CSA scheme with $k=2$ and with the same rates, with the inclusion of $R=3 / 5$, under the random code hypothesis. All distributions have been optimized under the constraint that the smallest local rate allowed for each user is $1 / 6$. For each distribution, the corresponding threshold $G^{*}$ and stability bound (righthand side of (18) are shown. Note that in the CSA case the threshold values are average values: Specific choices of the codes $\mathscr{C}_{h}$ may lead to thresholds $G^{*}$ larger than $\bar{G}^{*}$. 


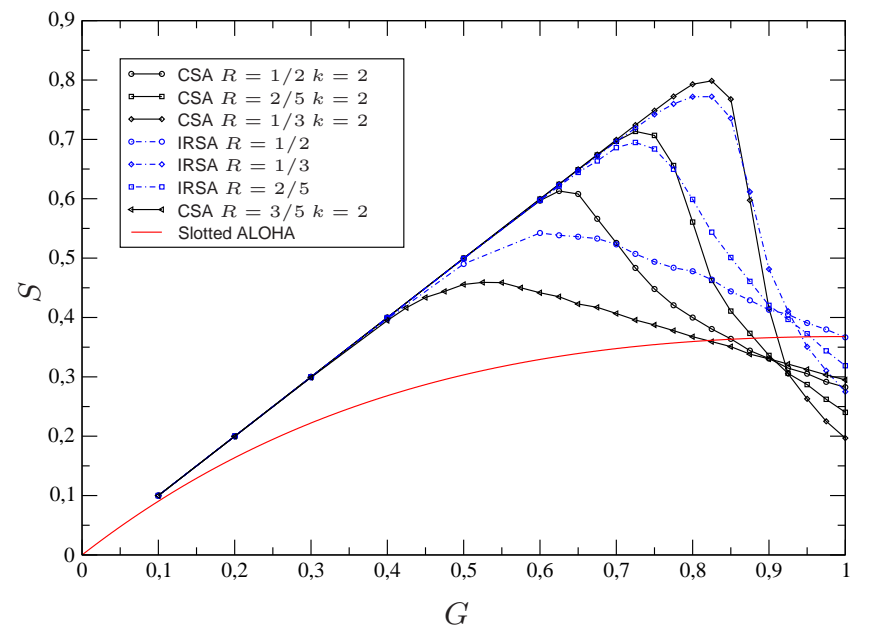

Fig. 2. Throughput versus the normalized offered traffic $G$ for IRSA and CSA schemes with p.m.f.'s in Table $\square$ The bursts of each CSA user are split into $k=2$ segments, so that $N_{\mathrm{CSA}}=2 N_{\mathrm{SA}} \cdot N_{\mathrm{CSA}}=1000, N_{\mathrm{SA}}=500$.

From Table \we see that CSA is capable to achieve better thresholds than IRSA for $R=1 / 2$ and $R=2 / 5$, while for the lowest rate $R=1 / 3$ IRSA exhibits a better threshold. Accordingly, the IRSA scheme seems to be preferable in the case of low rates $R$ (i.e., for higher values of the excess power $\Delta P$ ), while CSA is more interesting for higher values of $R$ (i.e., when a higher power efficiency is required). Note also that CSA allows to achieve values of the overall rate $R>1 / 2$, whereas only low rates $R \leq 1 / 2$ can be obtained from IRSA, unless some users transmit their burst in the MAC frame with no repetition. (In this latter case, however, no successful iterative IC can be guaranteed, so that we always have $G^{*}=0$.) For example, in Table \ an optimized CSA distribution of rate $R=3 / 5$ is reported. This distribution has no IRSA counterpart.

To validate our asymptotic analysis, we performed numerical simulations in the case of a finite number $M$ of users 6 In Fig. 2, the throughput curves of IRSA and CSA schemes with the probability profiles from Table \ are depicted as functions of the normalized offered traffic $G$. The throughput achieved by $\mathrm{SA}, S=G e^{-G}$, is also shown for reference. Note that in our simulations for the CSA case, we combined the p.m.f.'s $Q$ derived under the random code hypothesis with a specific choice of the component codes. In particular, we used linear block codes with the following generator matrices: $\mathbf{G}_{(3,2)}=[110,011]$, $\mathbf{G}_{(4,2)}=[1100,0111], \mathbf{G}_{(5,2)}=[11100,00111], \mathbf{G}_{(8,2)}=$ $[111100000,0111111], \mathbf{G}_{(9,2)}=$ [111110000, 011111111], $\mathbf{G}_{(12,2)}=[111111110000,0000011111111]$. To stay fair, we compared CSA $(k=2)$ and IRSA schemes for the same frame duration $T_{\mathrm{F}}$, which implies a number of slots $N_{\mathrm{CSA}}$ twice the number of slots $N_{\mathrm{SA}}$. Specifically, the simulations are for $N_{\mathrm{CSA}}=1000$ and $N_{\mathrm{SA}}=500$. For each value of $G$,

\footnotetext{
${ }^{6} \mathrm{Here}$, one shall consider that each segment has to be encoded via a physical layer error correcting code before transmission on the MAC channel, and that the physical layer code for CSA is $k$ times shorter than the corresponding code for IRSA. Thus, CSA may require working at slightly higher SNRs than IRSA, especially when short segments (and then short physical layer codes) are used. This aspect is not considered in this work.
}

$M$ can be obtained from (1). We observe a very good match between the asymptotic analysis and the simulations, the larger peak throughput of CSA than IRSA also for $R=1 / 3$ being essentially due to the specific choice of the component codes (recall that $\bar{G}^{*}$ is an average value).

\section{CONCLUSION}

Coded slotted ALOHA has been introduced as a new opportunity for high-throughput random access to the MAC channel. Density evolution equations for CSA have been derived, optimal CSA schemes designed for several rates and their performance for a finite number of users simulated. The new scheme is promising when power efficiency is required.

\section{REFERENCES}

[1] N. Abramson, "Multiple access in wireless digital networks," Proc. IEEE, vol. 82, no. 9, pp. 1360-1370, Sep. 1994.

[2] — "The ALOHA system - another alternative for computer communications," in Proc. of 1970 Fall Joint Computer Conf., vol. 37. AFIPS Press, 1970, pp. 281-285.

[3] L. G. Roberts, "ALOHA packet systems with and without slots and capture," ARPANET System Note 8 (NIC11290), Jun. 1972.

[4] C. Morlet, A. B. Alamanac, G. Gallinaro, L. Erup, P. Takats, and A. Ginesi, "Introduction of mobility aspects for DVB-S2/RCS broadband systems," IOS Space Communications, vol. 21, no. 1-2, pp. 5-17, 2007.

[5] G. Choudhury and S. Rappaport, "Diversity ALOHA - a random access scheme for satellite communications," IEEE Trans. Commun., vol. 31, no. 3, pp. 450-457, Mar. 1983.

[6] E. Casini, R. De Gaudenzi, and O. del Rio Herrero, "Contention resolution diversity slotted ALOHA (CRDSA): An enhanced random access scheme for satellite access packet networks." IEEE Trans. Wireless Commun., vol. 6, no. 4, pp. 1408-1419, Apr. 2007.

[7] O. del Rio Herrero and R. De Gaudenzi, "A high-performance MAC protocol for consumer broadband satellite systems," in Proc. of 27th AIAA Int. Communications Satellite Systems Conf. (ICSSC), Jun. 2009.

[8] G. Liva, "A Slotted ALOHA Scheme Based on Bipartite Graph Optimization," in Proc. of 2010 Int. ITG Conf. on Source and Channel Coding, Jan. 2010.

[9] — - "Graph-based analysis and optimization of contention resolution diversity slotted ALOHA," IEEE Trans. Commun., accepted for publication.

[10] M. Luby, M. Mitzenmacher, M. Shokrollahi, and D. Spielman, "Efficient erasure correcting codes," IEEE Trans. Inf. Theory, vol. 47, no. 2, pp. 569-584, Feb. 2001.

[11] T. J. Richardson, M. A. Shokrollahi, and R. L. Urbanke, "Design of capacity-approaching irregular low-density parity-check codes," IEEE Trans. Inf. Theory, vol. 47, no. 2, pp. 619-637, Feb. 2001.

[12] J. Massey, "The use of redundant packets in slotted-ALOHA-type random access systems," in Proc. of 1978 Int. Conf. on Information Sciences and Systems, Baltimore, MD, USA, Mar. 1978.

[13] A. Lam and D. Sarwate, "Time-hopping and frequency-hopping multiple-access packet communications," IEEE Trans. Commun., vol. 38, no. 6, pp. 875-888, Jun. 1990.

[14] E. Paolini, M. Fossorier, and M. Chiani, "Generalized and doubly generalized LDPC codes with random component codes for the binary erasure channel," IEEE Trans. Inf. Theory, vol. 56, no. 4, pp. 1651-1672, Apr. 2010.

[15] T. Helleseth, T. Kløve, and V. I. Levenshtein, "On the information function of an error-correcting code," IEEE Trans. Inf. Theory, vol. 43, pp. 549-557, Mar. 1997.

[16] A. Ashikhmin, G. Kramer, and S. ten Brink, "Extrinsic information transfer functions: Model and erasure channel properties," IEEE Trans. Inf. Theory, vol. 50, no. 11, pp. 2657-2673, Nov. 2004.

[17] E. Paolini, M. Fossorier, and M. Chiani, "Doubly-generalized LDPC codes: Stability bound over the BEC," IEEE Trans. Inf. Theory, vol. 55, no. 3, pp. 1027-1046, Mar. 2009.

[18] _ _ "Generalized and doubly-generalized LDPC codes with random component codes for the binary erasure channel," IEEE Trans. Inf. Theory, vol. 56, no. 4, pp. 1651-1672, Apr. 2010.

[19] K. Price, R. Storn, and J. Lampinen, Differential Evolution: A Practical Approach to Global Optimization. Berlin, Germany: Springer-Verlag, 2005 . 